\title{
Clinical Correlates of Insomnia in Patients with Persistent Post-Traumatic Headache Compared with Migraine
}

\section{Soo-Kyoung Kim}

Gyeongsang National University College of Medicine https://orcid.org/0000-0003-0994-8503

\section{Catherine D Chong}

Mayo Clinic Arizona

Gina Dumkrieger

Mayo Clinic Arizona

\section{Katherine Ross}

Phoenix VA Health Care System

\section{Visar Berisha}

Arizona State University

Todd J. Schwedt ( $\square$ Schwedt.Todd@mayo.edu )

https://orcid.org/0000-0002-7780-7086

\section{Research article}

Keywords: posttraumatic headache, migraine, traumatic brain injury, insomnia, sleep

Posted Date: April 2nd, 2020

DOI: https://doi.org/10.21203/rs.2.21982/v2

License: (1) This work is licensed under a Creative Commons Attribution 4.0 International License.

Read Full License

Version of Record: A version of this preprint was published at The Journal of Headache and Pain on April 15th, 2020. See the published version at https://doi.org/10.1186/s10194-020-01103-8. 


\section{Abstract}

Background Close associations between insomnia with other clinical factors have been identified in migraine, but there have been few studies investigating associations between insomnia and clinical factors in patients with persistent post-traumatic headache (PPTH). The study objective was to contrast the severity of insomnia symptoms in PPTH, migraine, and healthy controls, and to identify factors associated with insomnia in patients with PPTH vs. migraine. Methods In this cross-sectional cohort study, 57 individuals with PPTH attributed to mild traumatic brain injury, 39 with migraine, and 39 healthy controls were included. Participants completed a detailed headache characteristics questionnaire, the Migraine Disability Assessment Scale (MIDAS), Insomnia Severity Index (ISI), Hyperacusis Questionnaire (HQ), Allodynia Symptom Checklist, Photosensitivity Assessment Questionnaire, Beck Depression Inventory (BDI), State-Trait Anxiety Inventory, Post-Traumatic Stress Disorder (PTSD) checklist, Ray Auditory Verbal Learning Test, and the Trail Making Test A and B to assess headache characteristics, disability, insomnia symptoms, sensory hypersensitivities, and neuropsychological factors. Fisher's test and one-way ANOVA or Tukey's Honest Significant Difference were used to assess group differences of categorical and continuous data. Stepwise linear regression analyses were conducted to identify clinical variables associated with insomnia symptoms. Results Those with PPTH had significantly higher ISI scores $(16.7 \pm 6.6)$ compared to migraine patients $(11.3 \pm 6.4)$ and healthy controls $(4.1 \pm 4.8)(p<$ 0.001). For those with PPTH, insomnia severity was most strongly correlated with the BDI (Spearman's rho ( Common.EditSubmissionSteps. Transform. EquationText $)=0.634, p<0.01$ ), followed by Trait Anxiety (Common.EditSubmissionSteps.Transform.EquationText $=0.522, p<0.01$ ), PTSD ( Common.EditSubmissionSteps. Transform. EquationText $=0.505, p<0.01), \mathrm{HQ}($ Common.EditSubmissionSteps. Transform. EquationText $=0.469, p<0.01)$, State Anxiety ( Common.EditSubmissionSteps.Transform. EquationText $=0.437, p<0.01)$, and MIDAS scores ( Common.EditSubmissionSteps. Transform. EquationText $=0.364, p<0.01)$. According to linear regression models, BDI, headache intensity, and hyperacusis scores were significantly positively associated with insomnia severity in those with PPTH, while only delayed memory recall was negatively associated with insomnia severity in those with migraine. Conclusions Insomnia symptoms were more severe in those with PPTH compared to migraine and healthy control cohorts. Depression, headache intensity, and hyperacusis were associated with insomnia in individuals with PPTH. Future studies should determine the bidirectional impact of treating insomnia and its associated symptoms.

\section{Background}

Headache is one of the most prevalent symptoms following mild traumatic brain injury (mTBI) (1), (2). Post-traumatic headache (PTH) as currently defined by the International Classification of Headache Disorders, third edition (ICHD-3), is a secondary headache disorder that develops within seven days after a head injury, regaining consciousness following a head injury, or discontinuation of medications that impair ability to feel or report a headache following a head injury (3). PTH of more than three months' duration is classified as persistent PTH (PPTH) (3). PTH is the most common type of pain attributed to 
$\mathrm{mTBl}$, and more than one third of individuals with acute PTH have headache persistence and thus develop PPTH $(1,2,4-6)$.

Individuals with acute PTH and PPTH often have a constellation of physical, psychological, and cognitive post-TBI symptoms. Previous studies have shown that insomnia and other sleep disturbances result in a delayed PTH recovery $(7,8)$. Sleep is often disrupted following $\operatorname{mTBI}(9,10)$ and may cause or intensify post-TBI symptoms such as depression, anxiety, suicidality, irritability, fatigue, cognitive deficits, and pain (11-15).

Insomnia is also common in patients with migraine. The close association between insomnia and migraine has been reported in several studies (16-19). Poor sleep quality among patients with migraine is significantly associated with headache frequency and headache-related disability (20). Since PPTH is often phenotypically very similar to migraine $(2,21,22)$, we hypothesized that insomnia would associate with other symptoms similarly in those with PPTH vs. migraine (23-25).

The objective of this study was to compare the severity of insomnia symptoms in those with PPTH to migraine and healthy controls, and to identify the clinical factors associated with insomnia in patients with PPTH vs those with migraine.

\section{Methods}

This was a cross-sectional cohort study in which participants were prospectively enrolled from November 2015 until February 2018. The data reported within this manuscript were collected as part of a United States Department of Defense funded study that aimed to compare clinical characteristics and brain imaging findings amongst those with PPTH vs. migraine (25-27) .

\section{Subjects}

All study participants were between 18 and 65 years of age. Patients with migraine were enrolled from the Mayo Clinic Headache Center or were recruited via advertisements posted at the Mayo Clinic in Phoenix, Arizona. Patients with PPTH were enrolled from the Mayo Clinic Department of Neurology and from the Phoenix VA Health Care System. Healthy controls were recruited from the Phoenix area via advertisements and word-of-mouth. The Ohio State University TBI Identification Method was used to assess for a history of TBI (28). Patients who had PTH attributed to mTBI for at least three months were included in the PPTH group regardless of their mechanism of brain injury. Headache diagnoses were made by a certified headache specialist (TS) using ICHD-3 beta diagnostic criteria (29). Patients with PPTH were excluded if they had a personal history of moderate or severe TBI, migraine, or any other headache type prior to their injury (except infrequent episodic tension-type headaches). Those with migraine were excluded if they had a personal history of TBI. Healthy control subjects had no history of any headache type (except infrequent episodic tension-type headaches) and no history of TBI. 
This study was reviewed and approved by the Mayo Clinic Institutional Review Board, the Phoenix VA Health Care System Institutional Review Board, and the US Department of Defense Human Research Protection Office.

All participants provided written informed consent prior to study participation.

\section{Sleep questionnaire}

Symptoms of insomnia were assessed using the Insomnia Severity Index (ISI) questionnaire (30). The ISI is a 7-item self-report questionnaire that evaluates sleep onset, sleep maintenance, early morning awakening, sleep dissatisfaction, noticeability of sleep problems by others, degree of distress, and impact of sleep disturbance on daily functioning. Each of the ISI items is rated on a scale of 0-4; total ISI scores range between 0 to 28 with higher scores indicating greater insomnia severity. The ISI total raw score is interpreted as follows: 0-7=absence of insomnia; 8-14=sub-threshold insomnia; 15-21=clinical insomnia with moderate severity; 22-28=clinical insomnia with severe severity (30-32). Individuals answer the ISI questions based on their symptom during the last two weeks.

\section{Headache questionnaires}

Those with migraine and those with PPTH provided detailed information about their headaches including age at onset, headache duration, headache frequency, presence of aura, headache characteristics, localization, average headache intensity, associated features, comorbidity, and medical and family history, using a structured interview . Participants completed the Migraine Disability Assessment Scale (MIDAS) to assess headache-related disability during the prior 90 days (33).

\section{Hypersensitivity questionnaires}

To investigate sensory hypersensitivities, all subjects completed the following: 1) Hyperacusis Questionnaire (HQ) which consists of 14 items designed to assess auditory symptoms and sound sensitivity. Items are rated on a 4-point scale (scores 0-3), with higher scores indicating greater sensitivity (ranging from 0 to 42) (34). 2) Allodynia Symptom Checklist (ASC) is a 12-item questionnaire that assesses for symptoms of cutaneous allodynia during headaches. A total ASC score $\geq 3$ suggests the presence of cutaneous allodynia, with higher scores indicating greater severity (35), (36). 3) Photosensitivity Assessment Questionnaire (PAQ) which consists of 16 questions eight of which assessed for avoidance of light (photophobia) and 8 of which assessed for light-seeking behavior (photophilia). Only the eight items related to photophobia were included to determine the severity of the photophobia (ranging from 0 to 8$)(37,38)$.

\section{Mood-related questionnaires}

Mood-related questionnaires included the Beck Depression Inventory (BDI) for assessing depression (39), the State-Trait Anxiety Inventory for assessing anxiety (40), and the Post-traumatic Stress Disorder (PTSD) checklist in primary care (PC-PTSD) for assessing PTSD (41). The BDI includes 21 items, with 
higher total scores indicating greater symptoms of depression (39). The State-Trait Anxiety Inventory consists of 20 questions that assess how the individual feels "right now" and 20 questions assessing how the individual "generally feels". Higher total scores suggest greater levels of anxiety (40). The PCPTSD is the 4-item screen for PTSD, with a total score $\geq 3$ suggesting the presence of PTSD (41).

\section{Cognitive function evaluation}

The Trail Making Test (TMT, A and B) (42) and The Rey Auditory Verbal Learning Test (RAVLT) (43) were used to evaluate cognitive function. The TMT is a test of executive function, speed of processing, and attention(44). The RAVLT is an assessment of auditory verbal learning and memory. A list of 15 words is read aloud to the participant, and then the participant is immediately asked to recall as many words as they remember (43). After 30-minutes of interpolated testing, the participant is again asked to recall the words from the first list (delayed recall) (45). For the TMT and RAVLT, each participant's test score was converted to a z-score based on age-matched normative data $(42,43,45,46)$.

\section{Statistical Analyses}

Descriptive statistics were used to compare demographic data and headache characteristics among groups. Frequencies were used for description of categorical variables. Fisher's exact test was used to assess group differences for categorical data. For the comparisons of parametric data of two groups, Student's t-test was used for parametric variables. As standard diagnostic plots did not show the violation of any assumptions, non-parametric data was presented as mean \pm standard deviation. For the variables applicable to only PPTH and migraine groups, such as years with headache, one-way ANOVA was used to assess the difference in mean values between groups. Tukey's Honest Significant Difference (HSD) was used to assess mean differences among the three groups. This test performs simultaneous pairwise comparisons and controls family-wise error rate for multiple testing. . According to Tukey's HSD, age was not significantly different across the three groups. Thus, age was not corrected for in subsequent analyses. Spearman's rank correlations between independent variables and total ISI scores were calculated to assess the relationship between insomnia severity and clinical factors. A stepwise multiple linear regression analysis was conducted to identify clinical variables associated with insomnia symptoms. The total ISI score was the dependent variable, while the candidate independent variables included sex, headache characteristics, sensory hypersensitivity scores, scores on psychological questionnaires, and cognitive test results. Patients with full datasets were included in this analysis. . Statistical significance was set at $\mathrm{p}$-value $<0.05$, and $95 \%$ confidence intervals $(\mathrm{Cl})$ were reported as appropriate. All analyses were performed with SPSS 25.0 (Chicago, IL, USA) software.

\section{Results}

Subjects:

A total of 137 subjects were recruited, consisting of 59 subjects with PTTH, 39 subjects with migraine, and 39 healthy controls. Two PPTH subjects were excluded due to incomplete questionnaires, leaving 57 
PPTH subjects in this analysis. Of those with PPTH, 25 had mTBI due to blasts, 17 due to falls, 8 were sports-related, and 7 were caused by motor vehicle accidents. Thirteen subjects with PPTH had one lifetime $\mathrm{mTBI}, 33$ had $2-5$, and 11 had more than five mTBIs. Amongst those with PPTH, the clinical headache phenotypes were most similar with migraine for 41 , probable migraine like for 12 , and tensiontype headache like for 4 according to the ICHD-3 criteria. Demographic profiles and clinical characteristics are shown in Table 1. Average age of subjects was $39.1 \pm 10.6$, and $55.6 \%$ were male. Comparing the patients with PPTH to those with migraine and healthy control, there were no significant differences in age (mean age: $\mathrm{PPTH}=38.2 \pm 10.7$ years vs. migraine $=41.3 \pm 11.5$ years vs. healthy control $=38.3 \pm$ 9.5; $p=0.313)$ or sex (PPTH $=$ males $37 / 57(64.9 \%)$ vs. migraine $=16 / 39(41.0 \%)$ vs. healthy control $=$ $22 / 39(56.4 \%), p=0.072$ ) Pairwise comparisons did show differences in sex between PPTH and migraine $(p=0.024)$ but not between PPTH and healthy control $(p=0.522)$ or between migraine and healthy control $(p=0.257)$.

The PPTH group had a lower headache frequency than the migraine group (mean headache days/30-day month $=16.0 \pm 8.3$ vs. $20.5 \pm 6.3, p=0.005)$ and fewer mean years with headache $(11.0 \pm 8.8$ years vs. $24.9 \pm 14.6$ years, $p<0.001)$. There were not significant differences in mean total MIDAS scores $(64.2 \pm$ 57.4 in the PTTH group vs. $52.2 \pm 37.6$ in the migraine group, $(p=0.256)$ (Table 1).

Insomnia, hypersensitivity and mood related scores:

The mean ISI scores were significantly higher in the PPTH group compared with migraine and healthy control groups ( $16.7 \pm 6.6$ vs $11.3 \pm 6.4$ and $4.1 \pm 4.8, p<0.001$ ) (Fig. 1 ). The proportion of individuals with severe insomnia with a total ISI score $=22-28(26.3 \%$ vs $7.7 \%, p<0.001)$ and moderate insomnia with a total ISI score $=15-21(40.4 \%$ vs $25.6 \%, p<0.001)$ was significantly higher in the patients with PPTH than those with migraine (Fig. 2). The mean total score on the HQ (22.7 \pm 10.6 vs $14.5 \pm 9.1$ and $5.7 \pm 4.8, p<0.001$ ) was higher in the PPTH group compared to the migraine and healthy control group. Based on ASC scores $\geq 3,66.7 \%$ (38/57) of patients with PPTH and $66.7 \%(26 / 39)$ of those with migraine had cutaneous allodynia $(p=1)$. The mean total PAQ score of photophobia was not different in the PPTH group compared to the migraine group $(4.6 \pm 2.6$ vs $3.8 \pm 2.5, p=0.270)$.

The mean total BDI scores (PPTH: $17.8 \pm 9.4$ vs migraine $8.7 \pm 6.0$ and healthy control $2.1 \pm 3.6, p<$ 0.001 ) and state anxiety scores ( $38.8 \pm 13.2$ vs $33.4 \pm 9.1$ and $23.6 \pm 5.7, p=0.038$ and $p<0.001$ ) were significantly higher in the PPTH group compared to the migraine and healthy control groups. Trait anxiety scores ( $44.6 \pm 14.1$ vs $39.4 \pm 10.9$ and $26.5 \pm 7.4, p=0.079$ and $p<0.001)$ were significantly higher in the PPTH group compared to the healthy control group, but were not significantly higher in the PPTH group compared to the migraine group. Based on scores $\geq 3$ for the PTSD, $68.4 \%(39 / 57)$ of patients with PPTH registered as having PTSD compared to $5.1 \%(2 / 39)$ of those with migraine $(p<0.001)($ Table1).

\section{Cognitive evaluation:}

The results of cognitive tests are presented in Table 2. Z-scores were calculated for scores from each group using normative values. The TMT A mean raw score was $27.6 \pm 9.4$ (z-score 0.18 ) in the PPTH 
group vs $27.7 \pm 11.0$ ( $z$-score 0.13$)$ in the migraine group $(p=0.973)$ and $23.1 \pm 5.1$ (z-score 0.59$)$ for healthy controls $(p=0.126)$. The TMT B mean raw score was $69.5 \pm 26.7$ ( $z$-score 0.16$)$ in the PPTH group vs $65.7 \pm 29.6$ (z-score 0.35$)$ in the migraine group $(p=0.585)$ and $58.1 \pm 21.1$ (z-score 0.52$)$ for healthy controls $(p=0.153)$. Time to completion did not significantly differ by the groups for either TMT A or TMT B. The z-scores with mean raw immediate recall scores (PPTH: -1.22 (5.5 \pm 2.1$)$ vs migraine: $-0.67(6.3 \pm 1.6), p=0.087$, and healthy control: $-0.69(6.4 \pm 1.4), p=0.109)$ and delayed recall scores $(-1.01(8.9 \pm 4.2)$ vs $-0.32(10.2 \pm 3.0), p=0.065$ and $-0.34(10.4 \pm 2.9), p=0.075)$ of RAVLT in the PTTH group were not significantly different than those of the migraine group and healthy controls.

Clinical factors associated with insomnia:

Among the PPTH group, BDI scores exhibited the strongest correlation with insomnia severity scores (Spearman's rho ()$=0.634, p<0.01)$, followed by trait anxiety scores $(=0.522, p<0.01)$, PTSD scores ( $=0.505, p<0.01), \mathrm{HQ}$ scores $(=0.469, p<0.01)$, state anxiety scores $(=0.437, p<0.01)$, MIDAS scores ( $=0.364, p<0.01)$ and ASC scores $(=0.284, p<0.05)$. In the PPTH group, there were no significant associations between insomnia severity and cognitive function according to TMT A and B and immediate and delayed recall of the RAVLT. However, the migraine group showed a significant correlation between increased insomnia severity and poorer performance for delayed recall on the RAVLT $(=-0.390, p<0.05)$ (Table 3).

In the migraine group, the stepwise multiple regression analysis produced a model including one variable that explained $12.9 \%$ of insomnia severity variance. The delayed memory recall was the only parameter significantly associated with insomnia severity $(\beta=-0.359, p=0.025)$ in the migraine group. In the PPTH group, regression analyses produced a model with 3 variables explaining $51.7 \%$ of insomnia severity variance $\left(R^{2}=0.517\right.$; adjusted $\left.R^{2}=0.489, F=56.11\right)$. The total $B D I$ score $(\beta=0.555, p<0.001)$ was most strongly associated with insomnia severity, followed by headache intensity $(\beta=0.230, p=0.019)$, and $\mathrm{HQ}$ score $(\beta=0.220, p=0.047)$ (Table 4).

\section{Discussion}

In this study, we found that PPTH and migraine patients had more severe insomnia symptoms relative to a healthy control cohort. The severity of insomnia symptoms was greater in individuals with PPTH attributed to mTBI compared to those with migraine. In those with PPTH, depression, headache intensity, and hyperacusis were the strongest factors associated with insomnia severity. Amongst those with migraine, delayed memory recall was related to insomnia severity. Our finding suggests that insomnia is prevalent and more severe in PPTH patients than migraine patients; nevertheless the majority of PPTH patients in this study had migraine-like headaches. These results indicate that we need to assess for insomnia in patients with PPTH, a condition that could be easily overlooked if the clinician focuses attention entirely on the headache and associated symptoms. Although the directionality of the relationships between insomnia and these associated symptoms cannot be determined from this crosssectional study, it is possible that effective management of insomnia could relieve some of these 
associated symptoms and vice-versa. Different than PPTH, our study suggests that insomnia might affect delayed memory recall in patients with migraine. Therefore, insomnia might have different clinical implications in those with PPTH vs. migraine, and these implications should be properly considered according to the underlying headache type (47).

Headache and insomnia frequently co-occur, and are mutually interacting conditions following TBI. Prior studies demonstrated that 30 70\% of patients with TBI complained of sleep disturbance $(48,49)$, and that insomnia was a potential risk factor for PTH (7). Insomnia and other sleep disturbances resulted in a delayed PTH recovery and could lead to PPTH $(7,8)$. Mechanistically, it is possible that insomnia could affect the descending pain inhibitory control system and enhance pain perception in patients with PTH $(50,51)$. In fact, in our study there was a positive correlation between insomnia severity with headache intensity and with symptoms of cutaneous allodynia. A recent study proposed that the perivascular glymphatic exchange impairment caused by TBI and sleep disruption may impair the clearance of neuropeptides such as calcitonin gene-related peptide (CGRP) involved in the pathogenesis of PTH (11). However, there is still a lack of studies investigating mechanisms linking the persistence of PTH and sleep disturbance attributed to TBI. In our study, the high prevalence (66.3\%) and severity of insomnia in those with PPTH suggest that insomnia might be associated with the persistence of pain in PTH after mTBI.

The pain of PPTH could also contribute to and exacerbate insomnia. Although markers of headache burden, such as years with headache and headache frequency, were lower in the PPTH group compared to the migraine group, insomnia was more prevalent and more severe among those with PPTH. It is possible that those with more years of headache adapt to their headache symptoms, resulting in less impact on sleep over time. These results could also suggest that factors in addition to headache may be involved in insomnia of PPTH and migraine.

In our study, depression was the strongest predictor of insomnia in those with PPTH. Our results also demonstrated that mood alterations including depression, anxiety and PTSD symptoms were reported more frequently in those with PPTH than those with migraine and healthy controls. Depression, anxiety, PTSD and suicidality symptoms are common following $\mathrm{mTBI}(7,52)$. Those with PPTH following mTBI might have low serotonin levels, contributing to development of psychological symptoms and insomnia (53-56). In addition, headache and non-headache pain are common comorbid conditions in those with PPTH, contributing to sleep disturbances and mood alterations (57). Our study showed that insomnia is associated with depression and headache intensity in those with PPTH, though the cause-effect relationship is unclear.

Hyperacusis was significantly associated with insomnia in those with PPTH in our data. Hyperacusis is the condition of reduced tolerance or increased sensitivity to sound, and is characterized by excessive loudness, troublesomeness, anxiety, and pain when the person is exposed to sound $(58,59)$. Auditory symptoms including subjective hearing loss, tinnitus, and hyperacusis are common in those with $\mathrm{mTBl}$, and $67.3 \%$ of non-blast mTBI patients reported hyperacusis according to a recent patient-reported 
outcome measures study (60). Mechanisms underlying hyperacusis following mTBI are unknown, although proposed theories suggest a tonotopic reorganization and hyperexcitability of the auditory cortex involving a malfunction of 5-HT (61), a release of endogenous opioid peptides (62), and a decreased $\mathrm{GABA}_{A}$-mediated inhibition in the inferior colliculus (63). Many studies have shown that the sleep disturbance generated by hyperacusis is mediated by psychological factors $(58,64,65)$. Anxiety and hyperresponsiveness to every sound while one is trying to sleep could result in insomnia. Fortunately, a recent study demonstrated that prophylactic treatment of migraine is associated with improvements in hyperacusis, suggesting that hyperacusis might share a pathophysiologic basis with migraine (66). It is yet to be conclusively demonstrated whether effective treatment of PPTH is associated with reductions in hyperacusis.

In this study, one third of individuals with migraine reported symptoms of insomnia, though the sample size was small. Prior studies have demonstrated that individuals with migraine are at an increased risk of having insomnia, and individuals with insomnia have a higher risk of migraine compared to individuals without insomnia $(17,18,67,68)$. In our study, insomnia severity was associated with delayed memory recall in individuals with migraine. The close relationship between insomnia and cognitive dysfunction has been described in previous studies (69-71). Future studies with larger sample sizes are needed to further determine the effects of insomnia on memory impairment in individuals with migraine.

This is the first study to compare the severity of insomnia symptoms in individuals with PPTH vs. those with migraine. The identification of associations between symptoms of depression, hyperacusis, and headache intensity with symptoms of insomnia in those with PPTH yields relational insights into these common post-TBI symptoms. However, there are several limitations in our study. First, the cross-sectional design does not allow for determining the direction of relationships between insomnia and its associated symptoms. Second, in order to avoid issues with recall bias, the response period for the insomnia questionnaire was limited to the prior two weeks. Although it is likely that symptoms during that two week period are reflective of symptoms over a longer time-period, this cannot be confirmed. It is not known if the individuals in this study had insomnia prior to development of PPTH or migraine, or if insomnia developed after headache onset. Third, we didn't have objective sleep measures and information about other sleep related disturbance (e.g., obstructive sleep apnea, restless leg syndrome). Fourth, we did not evaluate additional auditory symptoms other than hyperacusis (e.g. tinnitus, hearing loss) and did not perform objective audiological investigations to identify or quantify them. Fifth, since many patients in our study were recruited from a specialty headache clinic, it is not clear if the results would be generalizable to the general population of people with PPTH

\section{Conclusion}

Although the vast majority of individuals with PPTH in this study had migraine-like headaches, insomnia was more severe among individuals with PPTH compared to those with migraine. Among those with PPTH, there were associations between insomnia symptom severity with symptoms of depression, headache intensity, and hyperacusis, relationships not shared by those with migraine. The frequency and 
severity of insomnia symptoms found in this study suggest that the clinician should assess patients with PPTH for insomnia. Although not conclusive from this cross-sectional study, the associations between insomnia with depression, headache intensity, and hyperacusis at least suggest that treatment of insomnia could lead to improvements in other symptoms associated with PPTH. Future studies should determine the bidirectional impact of treating insomnia and its associated symptoms.

\section{Abbreviations}

mTBI: mild traumatic brain injury; PTH: Post-traumatic headache; ICHD-3: the International Classification of Headache Disorders, third edition; PPTH: Persistent post-traumatic headache; HC: healthy controls; MIDAS: Migraine Disability Assessment Scale; ISI: Insomnia Severity Index; ASC: Allodynia Symptom Checklist; HQ: Hyperacusis Questionnaire; PAQ: Photosensitivity Assessment Questionnaire; BDI: Beck Depression Inventory; STATE: State Anxiety Inventory; PTSD: Post-traumatic Stress Disorder; PC-PTSD: The primary care Post-traumatic Stress Disorder; TMT: Trail Making Test; RAVLT: Rey Auditory Verbal Learning Test; SD: standard deviation; N/A: Not applicable; ANOVA: Analysis of variance

\section{Declarations}

\section{Acknowledgements}

The authors would like to thank all study participants, research coordinators and imaging technologists for their time and dedication to this project.

\section{Author Information}

Affiliations

Department of Neurology, Mayo Clinic, Phoenix, AZ, USA

Catherine D Chong, Gina Dumkrieger, and Todd J. Schwedt

Department of Neurology and Institute of Health Science, Gyeongsang National University College of Medicine, South Korea

Soo-Kyoung Kim

Phoenix VA Health Care System, Phoenix, AZ, USA

Katherine Ross

Arizona State University, Tempe, AZ, USA

Visar Berisha 


\section{Ethics approval and consent to participate}

Mayo Clinic Institutional Review Board, Phoenix VA Health Care System Institutional Review Board, and U.S. Department of Defense Human Research Protection Office approvals were obtained prior to initiation of study procedures. Each research participant underwent an informed consent process during which the potential risks and benefits of study participation were discussed.

\section{Consent for publication}

Not applicable.

\section{Availability of data and materials}

The dataset supporting the conclusions of this article is available via the Federal Interagency Traumatic Brain Injury Research (FITBIR) informatics system.

\section{Competing interests}

The authors declare that they have no competing interests.-- Todd J. Schwedt, MD, has served as a consultant for Alder, Allergan, Amgen, ATI, Aural Analytics, Avanir, Biohaven, Cipla, Click Therapeutics, Dr. Reddy's Laboratories, Eli Lilly, Equinox, Ipsen Bioscience, Nocira, Novartis, Promius Pharma, Second Opinion, Teva, and Xoc. He holds stock options in Aural Analytics, Nocira, and Second Opinion and has received research funding from Amgen.

\section{Funding}

This study was funded by the Office of the Assistant Secretary of Defense for Health Affairs, through the Peer Reviewed Medical Research Program under Award No. W81XWH-15-1-0286 and Award No. W81XWH-19-1-0534. Opinions, interpretations, conclusions, and recommendations are those of the authors and are not necessarily endorsed by the Department of Defense. The U.S. Army Medical Research Acquisition Activity, 820 Chandler Street, Fort Detrick MD 21702-5014 is the awarding and administering acquisition office.

\section{Authors' Contributions}

TJS conceived the project, participated in data analyses, participated in the manuscript writing process, read and approved the final manuscript. CDC conceived the project, participated in data analyses, participated in the manuscript writing process, read and approved the final manuscript. KSK participated in data analyses, participated in the manuscript writing process, read and approved the final manuscript. GD conceived the project, participated in data analyses, participated in the manuscript writing process, read and approved the final manuscript. KR participated in obtaining IRB approvals at the Phoenix VA Health Care System, developing CRFs, enrolling subjects, administering study questionnaires, entering data into the REDCap database, and publishing and presenting study results. VB conceived the project, 
participated in data analyses, participated in the manuscript writing process, read and approved the final manuscript.

\section{Corresponding author}

Correspondence to Todd Schwedt

\section{References}

1. Nampiaparampil DE. Prevalence of chronic pain after traumatic brain injury: a systematic review. JAMA. 2008;300(6):711-9.

2. Lucas S, Hoffman JM, Bell KR, Dikmen S. A prospective study of prevalence and characterization of headache following mild traumatic brain injury. Cephalalgia. 2014;34(2):93-102.

3. Olesen J. International Classification of Headache Disorders. Lancet Neurol. 2018;17(5):396-7.

4. Lew HL, Lin PH, Fuh JL, Wang SJ, Clark DJ, Walker WC. Characteristics and treatment of headache after traumatic brain injury: a focused review. Am J Phys Med Rehabil. 2006;85(7):619-27.

5. Hoffman JM, Lucas S, Dikmen S, Braden CA, Brown AW, Brunner R, et al. Natural history of headache after traumatic brain injury. J Neurotrauma. 2011;28(9):1719-25.

6. Theeler BJ, Erickson JC. Posttraumatic headache in military personnel and veterans of the iraq and afghanistan conflicts. Curr Treat Options Neurol. 2012;14(1):36-49.

7. Chaput G, Giguere JF, Chauny JM, Denis R, Lavigne G. Relationship among subjective sleep complaints, headaches, and mood alterations following a mild traumatic brain injury. Sleep Med. 2009;10(7):713-6.

8. Hou L, Han X, Sheng P, Tong W, Li Z, Xu D, et al. Risk factors associated with sleep disturbance following traumatic brain injury: clinical findings and questionnaire based study. PLoS One. 2013;8(10):e76087.

9. Mathias JL, Alvaro PK. Prevalence of sleep disturbances, disorders, and problems following traumatic brain injury: a meta-analysis. Sleep Med. 2012;13(7):898-905.

10. Kostyun RO, Milewski MD, Hafeez I. Sleep disturbance and neurocognitive function during the recovery from a sport-related concussion in adolescents. Am J Sports Med. 2015;43(3):633-40.

11. Piantino J, Lim MM, Newgard CD, lliff J. Linking Traumatic Brain Injury, Sleep Disruption and PostTraumatic Headache: a Potential Role for Glymphatic Pathway Dysfunction. Curr Pain Headache Rep. 2019;23(9):62.

12. DeKosky ST, Ikonomovic MD, Gandy S. Traumatic brain injury: football, warfare, and long-term effects. Minn Med. 2010;93(12):46-7.

13. Minen MT, Boubour A, Walia H, Barr W. Post-Concussive Syndrome: a Focus on Post-Traumatic Headache and Related Cognitive, Psychiatric, and Sleep Issues. Curr Neurol Neurosci Rep. 2016;16(11):100. 
14. Ponsford JL, Parcell DL, Sinclair KL, Roper M, Rajaratnam SM. Changes in sleep patterns following traumatic brain injury: a controlled study. Neurorehabil Neural Repair. 2013;27(7):613-21.

15. Madsen T, Erlangsen A, Orlovska S, Mofaddy R, Nordentoft M, Benros ME. Association Between Traumatic Brain Injury and Risk of Suicide. JAMA. 2018;320(6):580-8.

16. Uhlig BL, Engstrom M, Odegard SS, Hagen KK, Sand T. Headache and insomnia in population-based epidemiological studies. Cephalalgia. 2014;34(10):745-51.

17. Odegard SS, Engstrom M, Sand T, Stovner LJ, Zwart JA, Hagen K. Associations between sleep disturbance and primary headaches: the third Nord-Trondelag Health Study. J Headache Pain. 2010;11(3):197-206.

18. Lateef T, Swanson S, Cui L, Nelson K, Nakamura E, Merikangas K. Headaches and sleep problems among adults in the United States: findings from the National Comorbidity Survey-Replication study. Cephalalgia. 2011;31(6):648-53.

19. Vgontzas A, Pavlovic JM. Sleep Disorders and Migraine: Review of Literature and Potential Pathophysiology Mechanisms. Headache. 2018;58(7):1030-9.

20. Walters AB, Hamer JD, Smitherman TA. Sleep disturbance and affective comorbidity among episodic migraineurs. Headache. 2014;54(1):116-24.

21. Vgontzas A, Cui L, Merikangas KR. Are sleep difficulties associated with migraine attributable to anxiety and depression? Headache. 2008;48(10):1451-9.

22. Vargas BB, Dodick DW. Posttraumatic headache. Curr Opin Neurol. 2012;25(3):284-9.

23. Ashina H, Porreca F, Anderson T, Amin FM, Ashina M, Schytz HW, et al. Post-traumatic headache: epidemiology and pathophysiological insights. Nat Rev Neurol. 2019;15(10):607-17.

24. Schwedt TJ, Chong CD, Peplinski J, Ross K, Berisha V. Persistent post-traumatic headache vs. migraine: an MRI study demonstrating differences in brain structure. J Headache Pain. 2017;18(1):87.

25. Howard L, Dumkrieger G, Chong CD, Ross K, Berisha V, Schwedt TJ. Symptoms of Autonomic Dysfunction Among Those With Persistent Posttraumatic Headache Attributed to Mild Traumatic Brain Injury: A Comparison to Migraine and Healthy Controls. Headache. 2018;58(9):1397-407.

26. Chong CD, Berisha V, Chiang CC, Ross K, Schwedt TJ. Less Cortical Thickness in Patients With Persistent Post-Traumatic Headache Compared With Healthy Controls: An MRI Study. Headache. 2018;58(1):53-61 .

27. Dumkrieger G, Chong CD, Ross K, Berisha V, Schwedt TJ. Static and dynamic functional connectivity differences between migraine and persistent post-traumatic headache: A resting-state magnetic resonance imaging study. Cephalalgia. 2019;39(11):1366-81.

28. Corrigan JD, Bogner J. Initial reliability and validity of the Ohio State University TBI Identification Method. J Head Trauma Rehabil. 2007;22(6):318-29.

29. Headache Classification Committee of the International Headache S. The International Classification of Headache Disorders, 3rd edition (beta version). Cephalalgia. 2013;33(9):629-808. 
30. Bastien $\mathrm{CH}$, Vallieres A, Morin $\mathrm{CM}$. Validation of the Insomnia Severity Index as an outcome measure for insomnia research. Sleep Med. 2001;2(4):297-307.

31. Morin CM, Belleville G, Belanger L, Ivers $H$. The Insomnia Severity Index: psychometric indicators to detect insomnia cases and evaluate treatment response. Sleep. 2011;34(5):601-8.

32. Chen PY, Yang CM, Morin CM. Validating the cross-cultural factor structure and invariance property of the Insomnia Severity Index: evidence based on ordinal EFA and CFA. Sleep Med. 2015;16(5):598603.

33. Stewart WF, Lipton RB, Dowson AJ, Sawyer J. Development and testing of the Migraine Disability Assessment (MIDAS) Questionnaire to assess headache-related disability. Neurology. 2001;56(6 Suppl 1):S20-8.

34. Khalfa S, Dubal S, Veuillet E, Perez-Diaz F, Jouvent R, Collet L. Psychometric normalization of a hyperacusis questionnaire. ORL J Otorhinolaryngol Relat Spec. 2002;64(6):436-42.

35. Lipton RB, Bigal ME, Ashina S, Burstein R, Silberstein S, Reed ML, et al. Cutaneous allodynia in the migraine population. Ann Neurol. 2008;63(2):148-58.

36. Florencio LL, Chaves TC, Branisso LB, Goncalves MC, Dach F, Speciali JG, et al. 12 item allodynia symptom checklist/Brasil: cross-cultural adaptation, internal consistency and reproducibility. Arq Neuropsiquiatr. 2012;70(11):852-6.

37. Wu Y, Hallett M. Photophobia in neurologic disorders. Transl Neurodegener. 2017;6:26.

38. Choi JY, Oh K, Kim BJ, Chung CS, Koh SB, Park KW. Usefulness of a photophobia questionnaire in patients with migraine. Cephalalgia. 2009;29(9):953-9.

39. Beck AT, Steer RA, Ball R, Ranieri W. Comparison of Beck Depression Inventories -IA and -II in psychiatric outpatients. J Pers Assess. 1996;67(3):588-97.

40. Ramanaiah NV, Franzen M, Schill T. A psychometric study of the State-Trait Anxiety Inventory. J Pers Assess. 1983;47(5):531-5.

41. Prins A, Bovin MJ, Smolenski DJ, Marx BP, Kimerling R, Jenkins-Guarnieri MA, et al. The Primary Care PTSD Screen for DSM-5 (PC-PTSD-5): Development and Evaluation Within a Veteran Primary Care Sample. J Gen Intern Med. 2016;31(10):1206-11.

42. Perianez JA, Rios-Lago M, Rodriguez-Sanchez JM, Adrover-Roig D, Sanchez-Cubillo I, Crespo-Facorro $B$, et al. Trail Making Test in traumatic brain injury, schizophrenia, and normal ageing: sample comparisons and normative data. Arch Clin Neuropsychol. 2007;22(4):433-47.

43. Davis JJ, Millis SR, Axelrod BN. Derivation of an embedded Rey Auditory Verbal Learning Test performance validity indicator. Clin Neuropsychol. 2012;26(8):1397-408.

44. Tombaugh TN. Trail Making Test A and B: normative data stratified by age and education. Arch Clin Neuropsychol. 2004;19(2):203-14.

45. Schoenberg MR, Dawson KA, Duff K, Patton D, Scott JG, Adams RL. Test performance and classification statistics for the Rey Auditory Verbal Learning Test in selected clinical samples. Arch Clin Neuropsychol. 2006;21(7):693-703. 
46. Ivnik RJ, Malec JF, Tangalos EG, Petersen RC, Kokmen E, Kurland LT. The Auditory-Verbal Learning Test (AVLT): Norms for Ages 55 Years and Older. Psychological Assessment: A Journal of Consulting and Clinical Psychology. 1990;2(3):304-12.

47. Jaramillo CA, Eapen BC, McGeary CA, McGeary DD, Robinson J, Amuan M, et al. A cohort study examining headaches among veterans of Iraq and Afghanistan wars: Associations with traumatic brain injury, PTSD, and depression. Headache. 2016;56(3):528-39.

48. Ouellet MC, Savard J, Morin CM. Insomnia following traumatic brain injury: a review. Neurorehabil Neural Repair. 2004;18(4):187-98.

49. Sandsmark DK, Elliott JE, Lim MM. Sleep-Wake Disturbances After Traumatic Brain Injury: Synthesis of Human and Animal Studies. Sleep. 2017;40(5).

50. Lautenbacher S, Kundermann B, Krieg JC. Sleep deprivation and pain perception. Sleep Med Rev. 2006;10(5):357-69.

51. Onen SH, Alloui A, Gross A, Eschallier A, Dubray C. The effects of total sleep deprivation, selective sleep interruption and sleep recovery on pain tolerance thresholds in healthy subjects. J Sleep Res. 2001;10(1):35-42.

52. Scholten AC, Haagsma JA, Cnossen MC, Olff M, van Beeck EF, Polinder S. Prevalence of and Risk Factors for Anxiety and Depressive Disorders after Traumatic Brain Injury: A Systematic Review. J Neurotrauma. 2016;33(22):1969-94.

53. Lustberg L, Reynolds CF. Depression and insomnia: questions of cause and effect. Sleep Med Rev. 2000;4(3):253-62.

54. Adrien J. Neurobiological bases for the relation between sleep and depression. Sleep Med Rev. 2002;6(5):341-51.

55. Pace-Schott EF, Hobson JA. The neurobiology of sleep: genetics, cellular physiology and subcortical networks. Nat Rev Neurosci. 2002;3(8):591-605.

56. Pompili M, Gibiino S, Innamorati M, Serafini G, Del Casale A, De Risio L, et al. Prolactin and thyroid hormone levels are associated with suicide attempts in psychiatric patients. Psychiatry Res. 2012;200(2-3):389-94.

57. Lavigne G, Khoury S, Chauny JM, Desautels A. Pain and sleep in post-concussion/mild traumatic brain injury. Pain. 2015;156 Suppl 1:S75-85.

58. Tyler RS, Pienkowski M, Roncancio ER, Jun HJ, Brozoski T, Dauman N, et al. A review of hyperacusis and future directions: part I. Definitions and manifestations. Am J Audiol. 2014;23(4):402-19.

59. Fackrell K, Stratmann L, Gronlund TA, Hoare DJ, Hyperacusis Priority Setting Partnership Steering G. Top ten hyperacusis research priorities in the UK. Lancet. 2019;393(10170):404-5.

60. Knoll RM, Herman SD, Lubner RJ, Babu AN, Wong K, Sethi RKV, et al. Patient-reported auditory handicap measures following mild traumatic brain injury. Laryngoscope. 2019.

61. Marriage J, Barnes NM. Is central hyperacusis a symptom of 5-hydroxytryptamine (5-HT) dysfunction? J Laryngol Otol. 1995;109(10):915-21. 
62. Sahley TL, Musiek FE, Nodar RH. Naloxone blockade of (-)pentazocine-induced changes in auditory function. Ear Hear. 1996;17(4):341-53.

63. Szczepaniak WS, Moller AR. Effects of (-)-baclofen, clonazepam, and diazepam on tone exposureinduced hyperexcitability of the inferior colliculus in the rat: possible therapeutic implications for pharmacological management of tinnitus and hyperacusis. Hear Res. 1996;97(1-2):46-53.

64. Aazh H, Moore BCJ. Factors Associated With Depression in Patients With Tinnitus and Hyperacusis. Am J Audiol. 2017;26(4):562-9.

65. Aazh H, Baguley DM, Moore BCJ. Factors Related to Insomnia in Adult Patients with Tinnitus and/or Hyperacusis: An Exploratory Analysis. J Am Acad Audiol. 2019;30(9):802-9.

66. Abouzari M, Tan D, Sarna B, Ghavami Y, Goshtasbi K, Parker EM, et al. Efficacy of Multi-Modal Migraine Prophylaxis Therapy on Hyperacusis Patients. Ann Otol Rhinol Laryngol. 2019:3489419892997.

67. Odegard SS, Sand T, Engstrom M, Zwart JA, Hagen K. The impact of headache and chronic musculoskeletal complaints on the risk of insomnia: longitudinal data from the Nord-Trondelag health study. J Headache Pain. 2013;14:24.

68. Lucchesi LM, Speciali JG, Santos-Silva R, Taddei JA, Tufik S, Bittencourt LR. Nocturnal awakening with headache and its relationship with sleep disorders in a population-based sample of adult inhabitants of Sao Paulo City, Brazil. Cephalalgia. 2010;30(12):1477-85.

69. Drummond SP, Walker M, Almklov E, Campos M, Anderson DE, Straus LD. Neural correlates of working memory performance in primary insomnia. Sleep. 2013;36(9):1307-16.

70. Fortier-Brochu E, Morin CM. Cognitive impairment in individuals with insomnia: clinical significance and correlates. Sleep. 2014;37(11):1787-98.

71. Fulda S, Schulz H. Cognitive dysfunction in sleep disorders. Sleep Med Rev. 2001;5(6):423-45.

\section{Tables}




\begin{tabular}{|c|c|c|c|c|c|c|}
\hline & $\begin{array}{l}\text { PPTH } \\
(\mathrm{n}=57)\end{array}$ & $\begin{array}{l}\text { Migraine } \\
(\mathrm{n}=39)\end{array}$ & $\begin{array}{c}\mathrm{HC} \\
(\mathrm{n}=39)\end{array}$ & $\begin{array}{c}\text { Migraine vs. } \\
\text { PPTH } \\
p \text {-value } \\
\end{array}$ & $\begin{array}{c}\text { PPTH vs. } \\
\text { HC } \\
p \text {-value }\end{array}$ & $\begin{array}{c}\text { Migraine } \\
\text { vs. HC } \\
p \text {-value }\end{array}$ \\
\hline \multirow[t]{2}{*}{ Age, mean (SD) } & $38.2(10.7)$ & $41.3(11.5)$ & $38.3(9.5)$ & & & \\
\hline & & & & 0.329 & 0.997 & 0.433 \\
\hline \multirow[t]{2}{*}{ Sex (female/male) } & $20 / 37$ & $23 / 16$ & $17 / 22$ & & 0.522 & \\
\hline & & & & 0.024 & & 0.257 \\
\hline \multirow[t]{2}{*}{ Headache intensity, mean (SD) } & $6.0(1.7)$ & $6.0(1.5)$ & & & & \\
\hline & & & $\mathrm{N} / \mathrm{A}$ & 0.981 & $\mathrm{~N} / \mathrm{A}$ & $\mathrm{N} / \mathrm{A}$ \\
\hline $\begin{array}{l}\text { Headache duration, hours, mean } \\
\text { (SD) }\end{array}$ & $26.3(26.4)$ & $\begin{array}{c}27.6 \\
(22.6)\end{array}$ & $\mathrm{N} / \mathrm{A}$ & 0.805 & $\mathrm{~N} / \mathrm{A}$ & $\mathrm{N} / \mathrm{A}$ \\
\hline $\begin{array}{l}\text { Headache frequency, days/month, } \\
\text { mean (SD) }\end{array}$ & $16.0(8.3)$ & $20.5(6.3)$ & $\mathrm{N} / \mathrm{A}$ & $0.005 *$ & $\mathrm{~N} / \mathrm{A}$ & $\mathrm{N} / \mathrm{A}$ \\
\hline Years with headache, mean (SD) & $11.0(8.8)$ & $\begin{array}{c}24.9 \\
(14.6)\end{array}$ & $\mathrm{N} / \mathrm{A}$ & $<0.001 *$ & $\mathrm{~N} / \mathrm{A}$ & $\mathrm{N} / \mathrm{A}$ \\
\hline MIDAS, mean (SD) & $64.2(57.4)$ & $\begin{array}{c}52.2 \\
(37.6)\end{array}$ & $\mathrm{N} / \mathrm{A}$ & 0.256 & $\mathrm{~N} / \mathrm{A}$ & $\mathrm{N} / \mathrm{A}$ \\
\hline ISI, mean (SD) & $16.7(6.6)$ & $11.3(6.4)$ & $4.1(4.8)$ & $<0.001 *$ & $<0.001 *$ & $<0.001 *$ \\
\hline $\mathrm{HQ}$, mean (SD) & $22.7(10.6)$ & $14.5(9.1)$ & $5.7(4.8)$ & $<0.001^{*}$ & $<0.001 *$ & $<0.001 *$ \\
\hline Allodynia, n (\%) & $38(66.7 \%)$ & $\begin{array}{c}26 \\
(66.7 \%)\end{array}$ & $\mathrm{N} / \mathrm{A}$ & 1.00 & $\mathrm{~N} / \mathrm{A}$ & $\mathrm{N} / \mathrm{A}$ \\
\hline PAQ, mean (SD) & $4.6(2.6)$ & $3.8(2.5)$ & $1.1(1.5)$ & 0.270 & $<0.001 *$ & $<0.001 *$ \\
\hline BDI, mean (SD) & $17.8(9.4)$ & $8.7(6.0)$ & $2.1(3.6)$ & $<0.001 *$ & $<0.001 *$ & $<0.001 *$ \\
\hline State, mean (SD) & $\begin{array}{c}38.8 \\
(13.2)\end{array}$ & $33.4(9.1)$ & $\begin{array}{l}23.6 \\
(5.7)\end{array}$ & 0.038 & $<0.001^{*}$ & $<0.001 *$ \\
\hline Trait, mean (SD) & $44.6(14.1)$ & $\begin{array}{c}39.4 \\
(10.9)\end{array}$ & $26.5(6.9)$ & 0.079 & $<0.001 *$ & $<0.001^{*}$ \\
\hline PTSD, n (\%) & 39 (68.4\%) & $2(5.1 \%)$ & $2(5.1 \%)$ & $<0.001 *$ & $<0.001 *$ & 1.00 \\
\hline
\end{tabular}

Table 1. Subject demographics and clinical characteristics by group.

SD: standard deviation; N/A: Not applicable; PPTH: Persistent post-traumatic headache; HC: healthy controls; Headache duration: average hours of headache per attack; Headache intensity: average pain intensity of headache per attack; Headache Frequency: number of headache days per month; Years with headache: years lived with headache; MIDAS: Migraine Disability Assessment Scale; ISI: Insomnia Severity Index; ASC: Allodynia Symptom Checklist; HQ: Hyperacusis Questionnaire; PAQ: Photosensitivity Assessment Questionnaire; BDI: Beck Depression Inventory; State: State anxiety score; Trait: Trait anxiety score; PTSD: The primary care Post-traumatic Stress Disorder screen

(Statistical threshold used $=^{*} p<0.05$ ) 


\begin{tabular}{|c|c|c|c|c|c|c|c|c|c|}
\hline & \multicolumn{2}{|c|}{$\begin{array}{c}\text { PPTH } \\
(\mathrm{n}=57)\end{array}$} & \multicolumn{2}{|c|}{$\begin{array}{c}\text { Migraine } \\
(n=39)\end{array}$} & \multicolumn{2}{|c|}{$\begin{array}{c}\mathrm{HC} \\
(\mathrm{n}=39)\end{array}$} & \multirow{2}{*}{$\begin{array}{c}\text { Migraine vs. } \\
\text { PPTH } \\
p \text {-value }\end{array}$} & \multirow{2}{*}{$\begin{array}{c}\text { PPTH } \\
\text { vs. HC } \\
p \text {-value }\end{array}$} & \multirow{2}{*}{$\begin{array}{c}\text { Migraine } \\
\text { vs. HC } \\
p \text {-value }\end{array}$} \\
\hline & $\begin{array}{c}\text { Mean } \\
(\mathrm{SD})\end{array}$ & $\begin{array}{c}\text { Z- } \\
\text { score }\end{array}$ & $\begin{array}{l}\text { Mean } \\
\text { (SD) }\end{array}$ & $\begin{array}{c}z^{-} \\
\text {score }\end{array}$ & Mean (SD) & $\begin{array}{c}\text { Z- } \\
\text { score }\end{array}$ & & & \\
\hline \multicolumn{10}{|l|}{ TMT A } \\
\hline & $\begin{array}{l}27.6 \\
(9.4)\end{array}$ & 0.18 & $\begin{array}{c}27.7 \\
(11.0)\end{array}$ & 0.13 & $23.1(5.1)$ & 0.59 & 0.973 & 0.126 & 0.115 \\
\hline TMT B & $\begin{array}{c}69.5 \\
(26.7)\end{array}$ & 0.16 & $\begin{array}{c}65.7 \\
(29.6)\end{array}$ & 0.35 & $58.1(21.1)$ & 0.52 & 0.585 & 0.153 & 0.702 \\
\hline $\begin{array}{l}\text { Immediate } \\
\text { recall }\end{array}$ & $\begin{array}{c}5.5 \\
(2.1)\end{array}$ & -1.22 & $\begin{array}{c}6.3 \\
(1.6)\end{array}$ & -0.67 & $6.4(1.4)$ & -0.69 & 0.087 & 0.109 & 0.995 \\
\hline $\begin{array}{l}\text { Delayed } \\
\text { recall }\end{array}$ & $\begin{array}{c}8.9 \\
(4.2)\end{array}$ & -1.01 & $\begin{array}{l}10.2 \\
(3.0)\end{array}$ & -0.32 & $10.4(2.9)$ & -0.34 & 0.065 & 0.075 & 0.998 \\
\hline
\end{tabular}

Table 2. Mean raw and z-scores for cognitive tests. Results of Tukey HSD performed on z-scores.

SD: standard deviation; PPTH: Persistent post-traumatic headache; HC: healthy controls; TMT: Trail making test

\begin{tabular}{|c|c|c|c|}
\hline & & $\begin{array}{l}\text { PPTH } \\
(n=57)\end{array}$ & $\begin{array}{c}\text { Migraine } \\
(n=39)\end{array}$ \\
\hline \multirow[t]{5}{*}{ Headache } & Headache intensity & 0.235 & -0.073 \\
\hline & Headache duration & -0.116 & -0.190 \\
\hline & Headache frequency & 0.069 & 0.045 \\
\hline & Years with headache & -0.009 & -0.124 \\
\hline & MIDAS & $0.364^{* *}$ & 0.268 \\
\hline \multirow[t]{3}{*}{ Hypersensitivity } & $\mathrm{HQ}$ & $0.469 * *$ & 0.240 \\
\hline & ASC & $0.284^{*}$ & 0.197 \\
\hline & PAQ & 0.181 & -0.057 \\
\hline \multirow[t]{4}{*}{ Mood } & BDI & $0.634^{* *}$ & 0.308 \\
\hline & PTSD & $0.505^{* *}$ & 0.097 \\
\hline & State & $0.437^{* *}$ & 0.046 \\
\hline & Trait & $0.522^{* *}$ & 0.156 \\
\hline \multirow[t]{4}{*}{ Cognitive function } & TMT A & -0.132 & -0.041 \\
\hline & TMT B & -0.154 & -0.071 \\
\hline & Immediate recall & -0.149 & -0.180 \\
\hline & Delayed recall & -0.164 & $-0.390 *$ \\
\hline
\end{tabular}


Table 3. Spearman correlation coefficients between Insomnia Severity Index and clinical parameters

${ }^{*}$ Correlation is significant at the 0.05 level (2-tailed).

${ }^{* *}$ Correlation is significant at the 0.01 level (2-tailed).

PPTH: Persistent Post traumatic headache; MIDAS: Migraine Disability Assessment Scale; HQ: Hyperacusis Questionnaire; ASC: Allodynia Symptom Checklist; PAQ: Photosensitivity Assessment Questionnaire; BDI: Beck Depression Inventory; PTSD: Post-traumatic Stress Disorder; State: State anxiety score; Trait: Trait anxiety score; TMT: Trail making test

\begin{tabular}{|c|c|c|c|c|c|c|c|c|}
\hline & $\begin{array}{l}\text { Unsta } \\
\text { Coeffi }\end{array}$ & $\begin{array}{l}\text { dized } \\
\text { s }\end{array}$ & $\begin{array}{l}\text { Standardized } \\
\text { Coefficients }\end{array}$ & & $\mathrm{t}$ & $\overline{R^{2}}$ & \multirow{2}{*}{\multicolumn{2}{|c|}{$\begin{array}{c}\text { Adjusted } \\
R^{2}\end{array}$}} \\
\hline & & B & SE & $\beta$ & & & & \\
\hline \multirow[t]{2}{*}{ Migraine } & (Constant) & 10.652 & 1.01 & & 10.548 & 0 & 0.129 & 0.106 \\
\hline & Delayed recall & -1.996 & 0.852 & -0.359 & -2.343 & $0.025 *$ & & \\
\hline \multirow[t]{4}{*}{$\mathrm{PPTH}$} & (Constant) & 1.219 & 2.810 & & 0.434 & 0.666 & 0.517 & 0.489 \\
\hline & BDI & 0.390 & 0.076 & 0.555 & 5.133 & $<0.001^{* *}$ & & \\
\hline & $\begin{array}{l}\text { Headache } \\
\text { intensity }\end{array}$ & 0.906 & 0.376 & 0.230 & 2.411 & $0.019 *$ & & \\
\hline & HQ & 0.137 & 0.067 & 0.220 & 2.034 & $0.047 *$ & & \\
\hline
\end{tabular}

Table 4. Multiple stepwise linear regression analysis for association of insomnia severity scores

Dependent Variable: Insomnia Severity Index score $(* p<0.05, * * p<0.01)$

Note. Independent variables such as sex, headache pain intensity, headache duration, headache frequency, years w. headache, MIDAS, HQ, ASC, PAQ, BDI, PTSD, State anxiety score, Trait anxiety score, TMT A and B, Immediate recall, and Delayed recall were included as candidate variables.

PPTH: Persistent Post traumatic headache; MIDAS: Migraine Disability Assessment Scale; HQ: Hyperacusis Questionnaire; ASC: Allodynia Symptom Checklist; PAQ: Photosensitivity Assessment Questionnaire; BDI: Beck Depression Inventory; PTSD: Post-traumatic Stress Disorder; TMT: Trail making test

\section{Figures}


Fig 1.

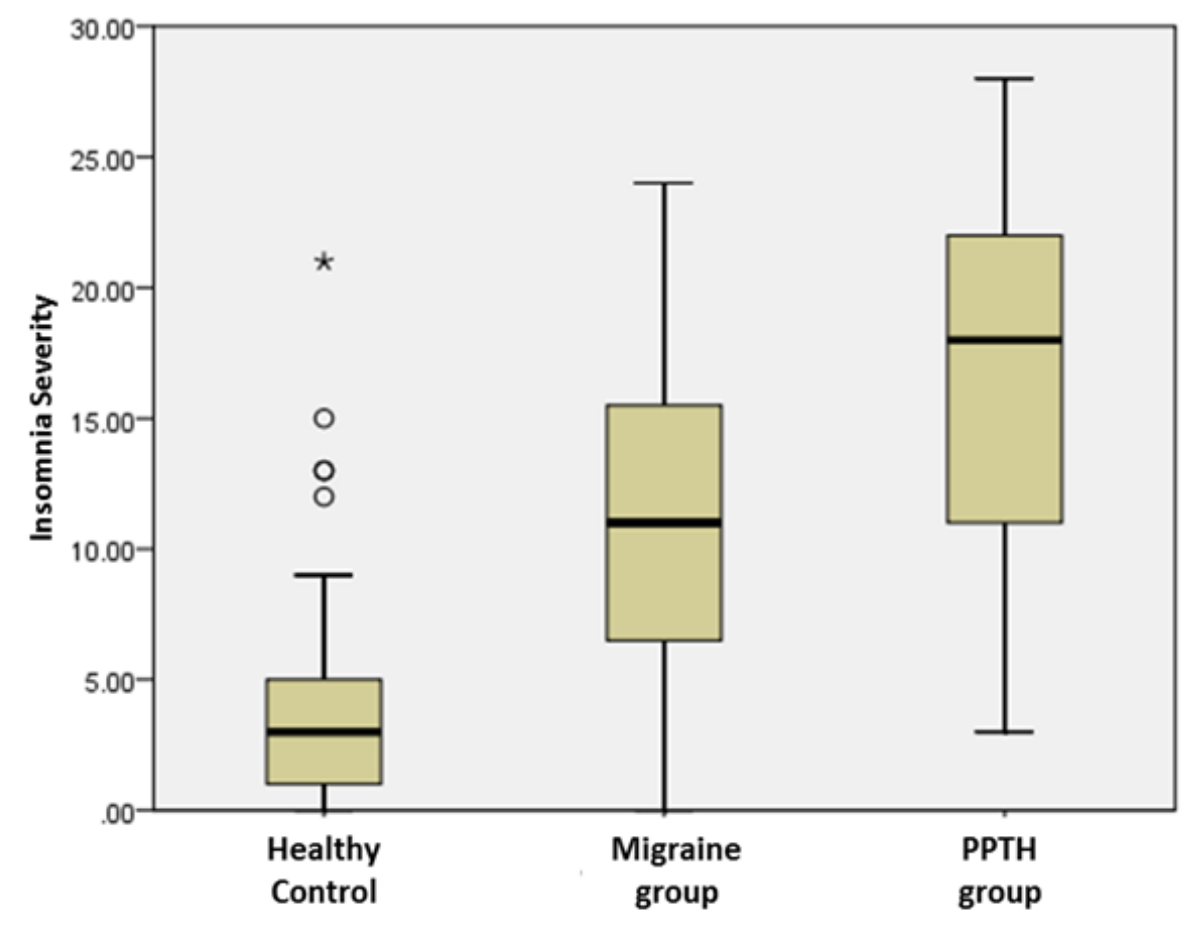

Figure 1

Insomnia Severity Index scores among groups PPTH: Persistent posttraumatic headache 
Fig 2.

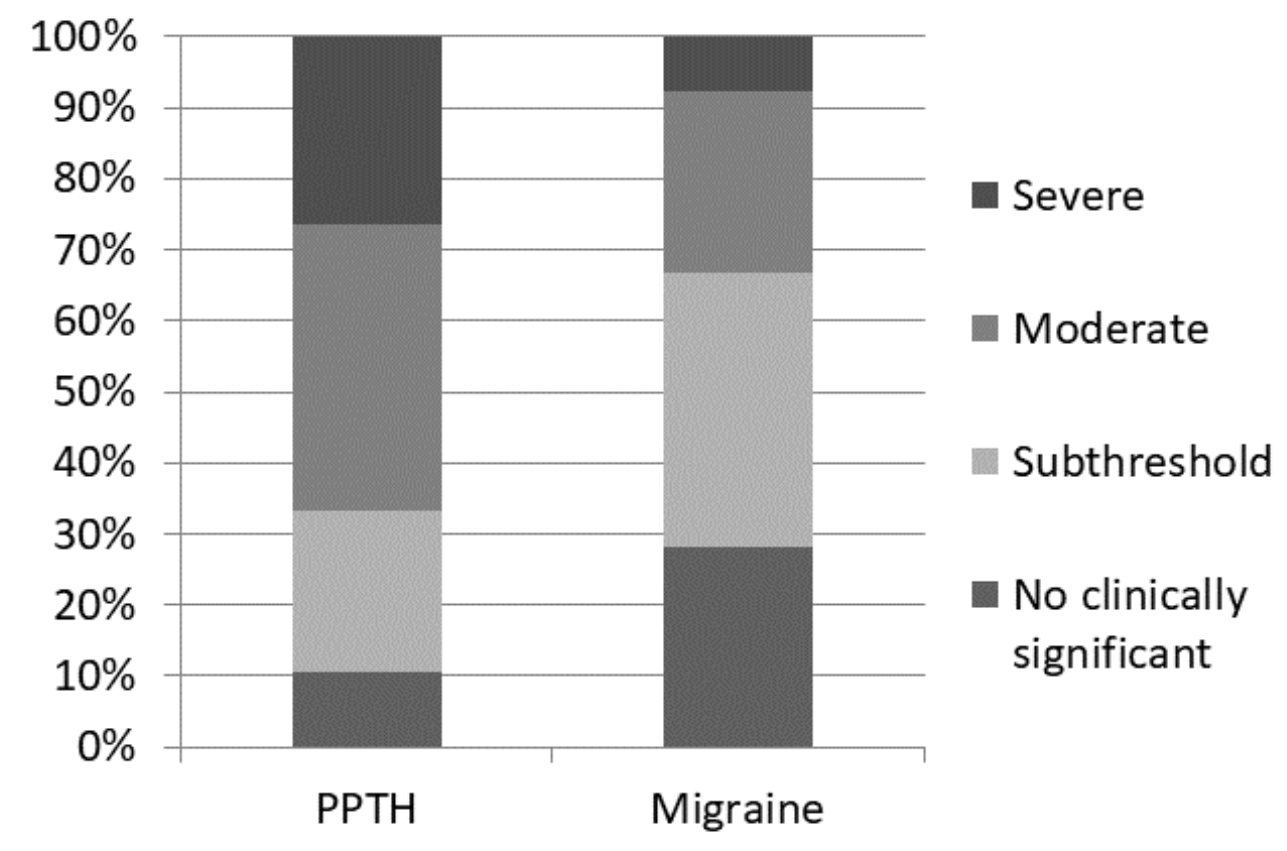

Figure 2

Distribution of insomnia severity in subjects with persistent post-traumatic headache and migraine PPTH: Persistent posttraumatic headache 